\title{
Adherence to and Persistence with Disease-Modifying Therapies for Multiple Sclerosis Over 24 Months: A Retrospective Claims Analysis
}

\author{
Gabriel Pardo · Elmor D. Pineda · Carmen D. Ng · Komal K. Bawa • \\ Daniel Sheinson · Nicole G. Bonine (D)
}

Received: October 8, 2021 / Accepted: December 21, 2021 / Published online: January 12, 2022

(c) The Author(s) 2022

\begin{abstract}
Introduction: We sought to assess adherence to and persistence with ocrelizumab (OCR) compared with other disease-modifying treatments (DMTs), by route of administration (RoA), for multiple sclerosis (MS) after 24 months in the United States.

Methods: This retrospective claims analysis of MS patients initiating a new DMT was conducted using the IBM MarketScan Commercial and Medicare Supplemental databases between April 2016 and December 2019. Continuous enrollment of $\geq 12$ months before and up to 24 months after initiating the index DMT was required. Adherence was assessed based on proportion of days covered (PDC) in the followup period with values $\geq 80 \%$ considered adherent. Persistence was defined as no evidence of switching to another DMT or no gap $\geq 60$ days in DMT coverage.
\end{abstract}

Supplementary Information The online version contains supplementary material available at https:// doi.org/10.1007/s40120-021-00319-3.

G. Pardo

Oklahoma Medical Research Foundation, Oklahoma City, OK, USA

E. D. Pineda $(\bowtie) \cdot$ C. D. Ng · K. K. Bawa ·

D. Sheinson - N. G. Bonine

Genentech, Inc., 1 DNA Way, South San Francisco,

CA 94080, USA

e-mail: pineda.elmor@gene.com
Results: A total of 1710 patients with $\geq 24$ months of follow-up (OCR, $n=524$; oral, $n=701$; injectable, $n=365$; other intravenous [IV], $n=120$ ) were included. Patients initiating OCR had higher adherence $(80 \%$ vs. $55 \%, 35 \%$, and $54 \%$ for oral, injectable, and other IV, respectively) and persistence (75\% vs. $54 \%, 33 \%$, and $55 \%$, respectively) at 24 months. Relative risks (RRs) of 24-month non-adherence for those initiating orals, injectables, and other IVs were 2.2 (95\% CI, 1.7-2.9), 3.0 (95\% CI, 2.2-4.0), and 2.2 (95\% CI, 1.5-3.3), respectively, compared to those initiating OCR. Similarly, patients receiving orals, injectables, and other IVs had RR of 1.9 (95\% CI, 1.4-2.4), 2.5 (95\% CI, 1.9-3.4), and 1.8 (95\% CI, 1.2-2.6) for 24-month discontinuation, respectively. Similar patterns were observed at 12 and 18 months.

Conclusions: Patients initiating OCR in a realworld setting achieved higher rates of adherence and persistence at 24 months compared with those initiating other DMTs, consistent with published literature showing similar results at 12 and 18 months. Optimizing medication adherence and persistence is fundamental to MS care, so clinicians should consider all elements of DMTs that may improve compliance.

Keywords: Multiple sclerosis; MS; Adherence; Persistence; Ocrelizumab 


\section{Key Summary Points}

Multiple sclerosis (MS) is a progressive condition that results in worsening neurological deficits over the disease course; non-adherence and nonpersistence with MS disease-modifying treatments (DMTs) has been associated with increased relapse rates, worse clinical outcomes, reduced healthcare quality of life, and higher medical care costs.

This retrospective claims analysis included 1710 patients with MS who received ocrelizumab (OCR) or other MS DMTs via oral, injectable, or other intravenous (IV) routes of administration (RoAs) to compare adherence and persistence.

At 24 months, the index OCR group achieved higher adherence and persistence rates than those who initiated MS DMTs via other RoAs.

At 24 months, $80 \%$ of patients initiating OCR were adherent compared with those initiating oral (55\%), injectable (35\%), and other IV medications (54\%); the mean proportion of days covered across groups was $88 \%, 70 \%, 56 \%$, and $67 \%$ for OCR, oral, injectable, and other IV, respectively; at 24 months, those initiating other DMTs were 2.2 to 3 times more likely to be non-adherent and 1.9 to 2.5 more likely to discontinue therapy compared to those initiating OCR.

The results from this analysis demonstrated that patients initiating OCR in a real-world setting achieved higher rates of adherence and persistence at 24 months compared with those initiating MS DMTs administered through other RoAs.

\section{INTRODUCTION}

Multiple sclerosis (MS), typically diagnosed between the third and fifth decades of life, is a progressive condition that results in worsening neurological deficits over the disease course [1]. The estimated prevalence of MS shows that while over 700,000 people in the US are affected, there is a disproportionate impact on women, with approximately a 3:1 female: male ratio [2]. For relapsing-remitting MS, the most common form of MS, more than 15 unique disease-modifying therapies (DMTs) are currently available in the United States to reduce disease activity [3]. MS is most commonly associated with physical limitations that result from relapses, but it also manifests with cognitive dysfunction, mood alteration, and reduced quality of life [4-6].

Understanding that medications can only have their desired effect when taken as prescribed, adherence to and persistence with therapy are vital components to slowing disease progression and decreasing the frequency of relapses. Specifically, as adherence is a known issue for people with MS, comprehensive efforts to increase compliance are thought to improve outcomes [7-9]. As such, clinicians have several options available to personalize treatment regimens that account for factors such as patient preference, route of administration (RoA), lifestyle, efficacy, tolerability, and adverse events $[7,10]$. Additionally, as MS affects people in their prime, following a definitive diagnosis, clinical practice guidelines support the early initiation of DMTs to optimize the impact, with several recommendations grounded in evaluating barriers and improving the adherence to DMTs [7].

Non-adherence and non-persistence with MS DMTs have been associated with increased relapse rates, worse clinical outcomes, reduced health-related quality of life, and higher medical care costs [11-16]. A recent analysis by Burks et al. [13] found adherence to oral and injectable DMTs significantly reduced the chances of relapse, hospitalization, and emergency visits at 1 year, thereby decreasing the annual medical care costs by $\$ 5816$ per patient. 
Another analysis by Yermakov et al. [14] found that an increase in adherence significantly decreased the number of workdays lost and hospital visits, in addition to direct and indirect costs. Furthermore, patients who were not persistent have also been found to be at increased risk of relapses, have increased health-related costs, and have a higher likelihood of inpatient admissions or emergency department visits $[17,18]$.

Research has shown that a variety of factors can influence MS treatment adherence, such as type of DMT, tolerability and perceived efficacy of DMT, age, out-of-pocket costs, socioeconomic status, and RoA [11, 19, 20]. A literature review of 24 studies found that currently available injectable DMTs showed modest adherence (weighted mean adherence ranged between 41 to $88 \%$ ) [11]. The weighted mean adherence was greater for the DMTs administered less frequently (once weekly, 69.4\%) compared with those with more frequent administration (three times per week, 58.4\%; daily, 56.8\%). On a related note, while oral DMTs on average have improved adherence over injectable therapies, Johnson et al. [12] found that adherence and persistence were also modest at $53-75 \%$ and $50-74 \%$, respectively. A systematic review similarly found that approximately $20 \%$ of patients were non-adherent and approximately 25\% discontinued oral DMTs before 1 year [21]. In addition, those who missed DMT doses were more likely to miss future doses [22]. Therefore, any factors likely to increase adherence, and thereby persistence, should be considered when initiating a DMT.

A recent study found that patients initiating ocrelizumab (OCR) had superior adherence and persistence at 12 and 18 months compared with patients who initiated DMTs administered through other routes [23]. OCR was approved by the US Food and Drug Administration (FDA) in 2017 and is currently the only therapy indicated for both relapsing and primary progressive MS. The OCR twice-yearly dosing schedule was hypothesized to increase compliance as it offers less frequent dosing compared with most other marketed DMTs. As the real-world evidence comparing OCR adherence and persistence with those of other DMTs is still limited, the objective of this study was twofold: (1) extend the analysis out to 24 months to explore these outcomes longer term and (2) confirm if the OCR adherence and persistence rates were consistent with the results from the previous study.

\section{METHODS}

\section{Data Source}

The retrospective cohort analysis was conducted using the IBM MarketScan Commercial and Medicare Supplemental claims databases. The MarketScan database is composed of administrative claims from large employers and health plans with de-identified data on medical and pharmacy claims for 273 million members from over 120 US employers and 40 contributing health plans [24]. The commercial database, which is the largest component of MarketScan, consists of the population under 65 years of age and their spouses and dependents, whereas the Medicare database consists of retirees covered by previous employers. Our analyses used inpatient and outpatient claims, diagnoses, and procedures based on the International Classification of Diseases, Ninth/Tenth Revision, Clinical Modification (ICD-9/10-CM), current procedural terminology (CPT) codes, and the healthcare common procedure coding system (HCPCS) from these databases.

Institutional review board approval and patient consent were not applicable as patient identifiers were absent from the secondary data used in this analysis. The data sets analyzed during the current study are not publicly available, as it is proprietary information.

\section{Patient Selection}

Patients with MS aged $\geq 18$ years who initiated an FDA-approved DMT between April 2017 and December 2018 were identified. The index date corresponded with the first use of a DMT in this period that had not already been prescribed or administered in the prior year. The start of the DMT initiation period corresponded with the 


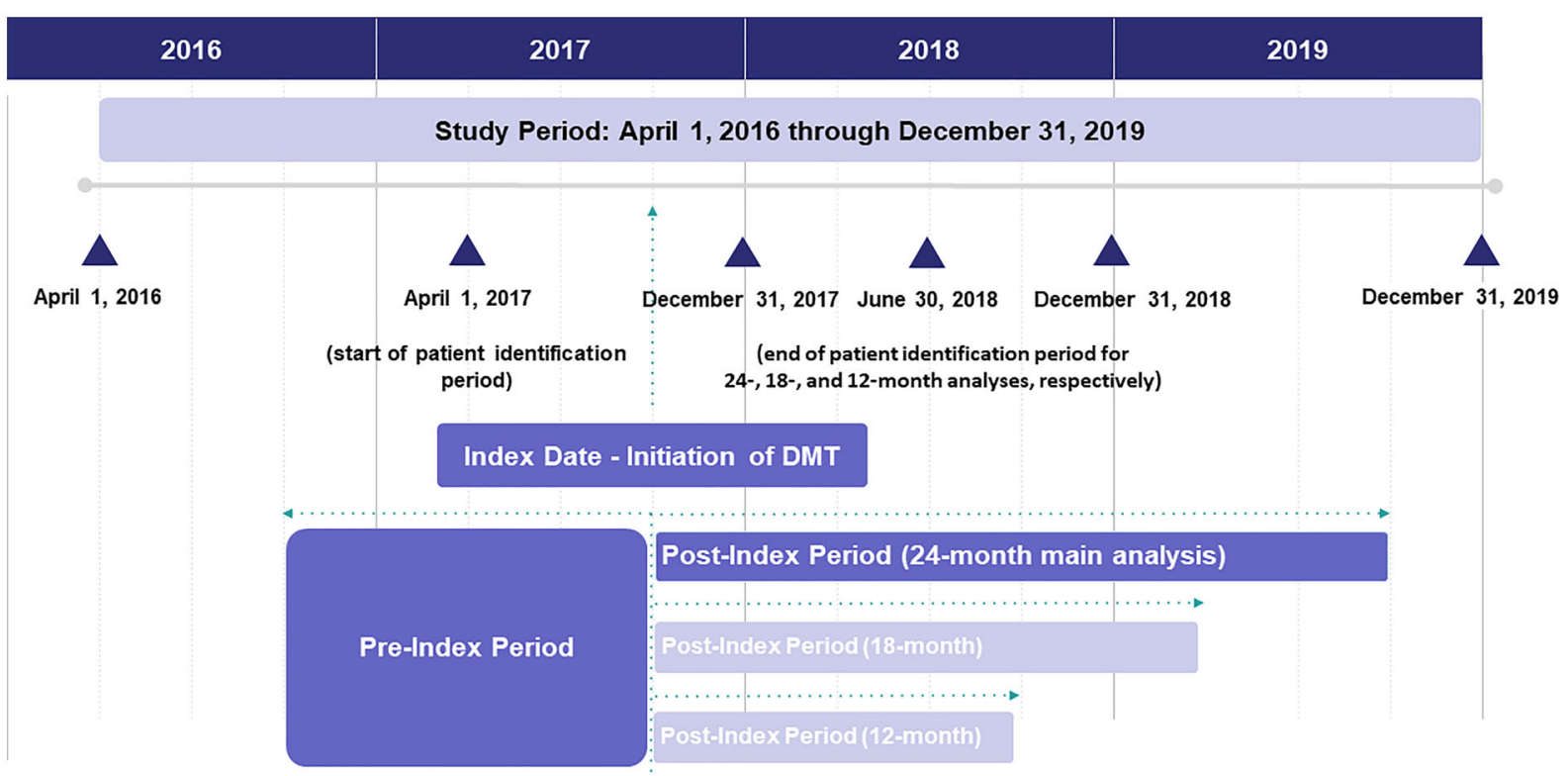

Fig. 1 Patient selection timeline. $D M T$ disease-modifying therapy

OCR FDA approval in the United States (March $28,2017)$. One year of continuous enrollment prior to the index date was required to adequately control for pre-index patient and disease characteristics (thus, the study period started on April 1, 2016). The main analysis required 24 months of continuous enrollment after the index date to measure outcomes; since claims were only available through December 31, 2019 (end of the study period), the period for DMT initiation in the 24-month main analysis ended on December 31, 2017. Note that we performed these analyses using the 12and 18-month outlooks on adherence and persistence as sensitivity analyses as well, which would vary the end of the DMT initiation period (June 30, 2018 for 18-month and December 31, 2018 for 12-month) (Figs. 1 and 2).

Patients were also required to have $>2$ prescriptions or administrations of the index DMT, thereby excluding one-time fills or administrations from the analysis. For OCR specifically, this requirement was met if there was evidence of the first split (loading) dose, which we defined as the first two intravenous (IV) infusions between 13 and 21 days apart and no subsequent dose within 100 days after the second infusion. Patients receiving OCR were identified by HCPCS J/C code (J2350, C9494),
National Drug Code Directory lists the NDC for ocrelizumab as 50242-150-01, or by satisfying all of the following criteria:

1. Miscellaneous HCPCS J codes (J3490, J3590, J9999, C9399) on or after April 1, 2017, that have CPT codes $(96413,96415,96365$, 96366) indicating IV infusion procedures within \pm 1 day.

2. An MS diagnosis on the same day as any identified miscellaneous HCPCS J code or any MS DMT use in the year prior to the earliest identified miscellaneous HCPCS J code.

3. No MS DMT use (other than OCR) on or within 6 months after the earliest identified miscellaneous HCPCS J code.

The criteria above are based on a published algorithm to identify OCR use before $\mathrm{J}$ and $\mathrm{C}$ codes were assigned [25].

Treatment groups were defined by the RoA: (1) OCR; (2) oral: teriflunomide, dimethyl fumarate, and fingolimod; (3) injectable (intramuscularly or subcutaneously): interferon $\beta 1 \mathrm{a} / \mathrm{b}$ and glatiramer acetate; (4) other IV: natalizumab. Patients receiving mitoxantrone were excluded due to insufficient sample size. Those receiving alemtuzumab were excluded as it is limited to a varying number of infusion courses, 


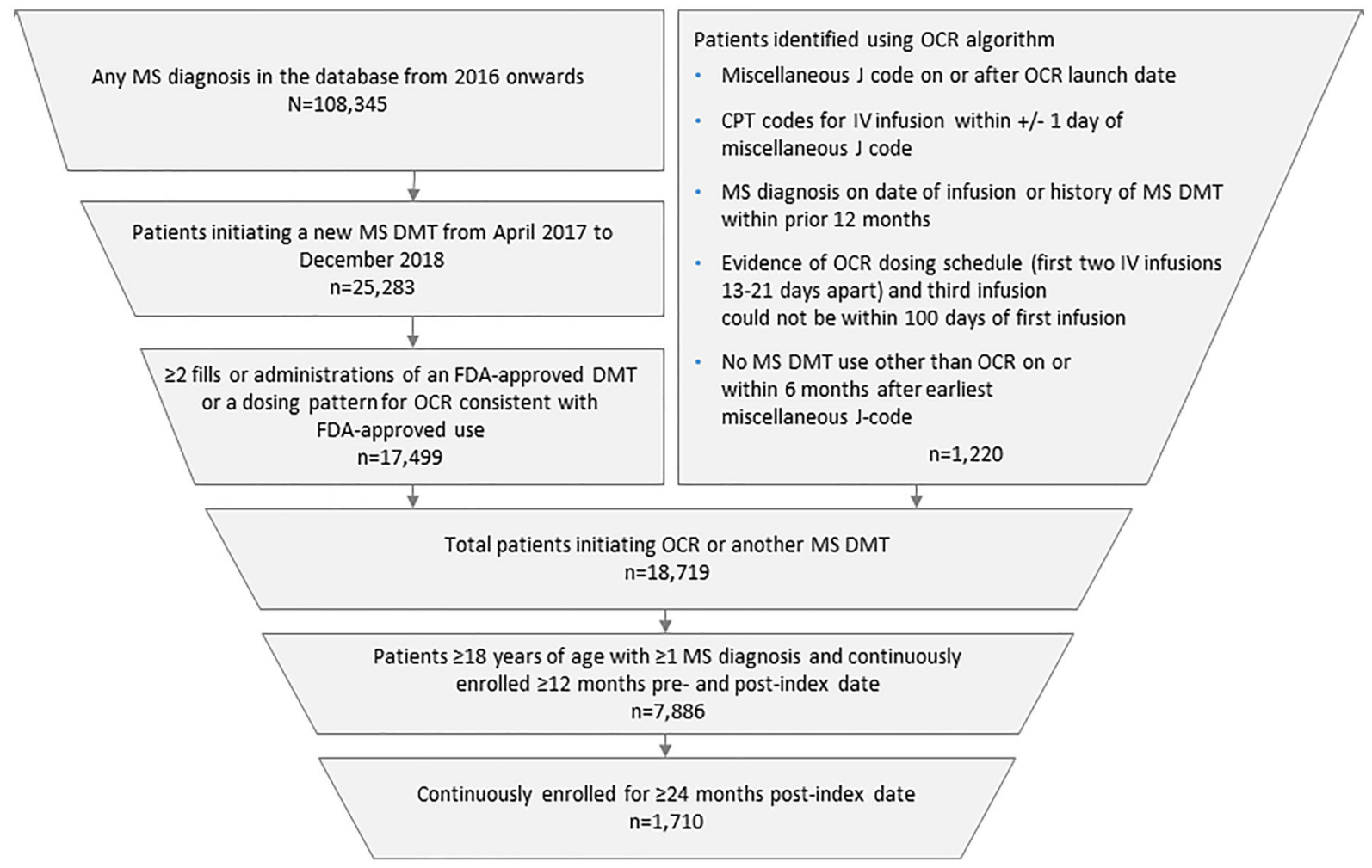

Fig. 2 Patient selection flow chart and patient counts. $C P T$ current procedural terminology, DMT disease-modifying therapy, $F D A$ US Food and Drug Administration,

and thus measuring adherence was not feasible. Off-label DMTs (e.g., rituximab) were also excluded due to the uncertainty of their indicated use within the data.

The primary analysis required $\geq 24$ months of continuous enrollment following the index date that corresponded to an index date cutoff of December 31, 2017. In sensitivity analyses, we evaluated outcomes of interest while requiring only 12 and 18 months of continuous enrollment following the index date, which allowed for the inclusion of patients initiating an MS DMT in 2018.

\section{Study Measures}

Patient demographics were measured at the index date. Clinical and disease characteristics, along with DMT usage, were based on pharmacy and medical claims during the year prior to the index date. Covariates included age, sex, region,
$I V$ intravenous, $M S$ multiple sclerosis, NDC National Drug Code, OCR ocrelizumab

payer, plan type, Charlson Comorbidity Index (CCI), prior experience of a relapse event, prior DMT use, and various MS symptoms. The MS symptoms included were based on previous MS claims analyses [23, 26] (Supplementary Table 1). Experience of a relapse event was identified in the pre-index year as an inpatient visit with a principal MS diagnosis or an outpatient visit with an MS diagnosis and a highdose oral steroid prescription (i.e., $\geq 500 \mathrm{mg} /$ day prednisone equivalent), injectable steroid, or plasma exchange/immunoglobulin administration within 30 days of the outpatient visit $[26,27]$.

\section{Study Outcomes}

Adherence was calculated using the proportion of days covered (PDC) measure, which determines the number of days covered by the DMT out of a fixed length of time (i.e., 12, 18, and 
24 months in this study). A PDC of $\geq 80 \%$ is deemed adherent for chronic diseases [28].

$\begin{aligned} \text { PDC }= & \left(\frac{\text { Number of covered days in the period }}{\text { Number of days in the period }}\right) \\ & \times 100 \%\end{aligned}$

Persistence was defined as no gap in supply $\geq 60$ days during the post-index period. Patients who had evidence of any other DMT during this time were also considered not persistent (switched).

For oral and injectable medications, the days of supply were determined directly from claims. For OCR and other IV medications, supply was 182 days and 28 days, respectively, from the start of each dose; this is based on each DMT's labeled dosing schedule. In calculations of both adherence and persistence, overlapping days of supply were accounted for by adding the extra days to the supply of the next dose. This adjustment was made to the days of supply for oral and injectable medications, while no adjustments for early administrations were made for OCR and other IV drugs.

Each day during the period was considered covered or not covered by drug supply depending on the number of days supplied from the previous fill or administration. After evidence of a switch to a different DMT, the patient was considered to have discontinued the index DMT.

All outcomes were assessed over a 12-, 18-, and 24-month follow-up period for all patients with at least that many months of continuous health plan enrollment. The primary analysis was conducted using the 24-month cohort data with secondary analyses completed for the 12 and 18-month cohorts.

\section{Statistical Analysis}

Continuous data are summarized as mean \pm $\mathrm{SD}$, and categorical data are presented descriptively as frequency and percentage. Patient disease characteristics and demographics were tabulated by treatment group (OCR, oral, injectable, and other IV). The mean crude PDC and proportion adherent and persistent at 12 ,
18 , and 24 months were reported by treatment group. Relative risks of non-adherence (PDC < $80 \%$ ) and discontinuation in the OCR vs. other DMT groups were estimated using a multivariable Poisson regression model (adjusted for the study measures described above). Using $R$ package emmeans, the relative risk (RR) of discontinuation for OCR compared with each RoA group was estimated [30]. Confidence intervals and $p$ values were adjusted for multiple comparisons by using an approximation to the Dunnett method [30].

All tests of statistical significance were two sided, and $p$ values $<0.05$ were considered statistically significant. The analyses were conducted using R version 3.5.3 (R Foundation for Statistical Computing, Vienna, Austria).

Various sensitivity analyses were conducted to ensure consistency in the results and recognize uncertainty. The first sensitivity analysis excluded all patients who initiated OCR in 2017 (for 12- and 18-month analyses), as these patients had a high likelihood of being identified via the OCR algorithm, which was used to identify OCR patients before the J or C code was established. This sensitivity check would highlight any misclassification of patients due to the OCR algorithm. Secondly, negative binomial models were used as an alternative to the Poisson models as a robustness check.

\section{RESULTS}

A total of 1710 patients receiving nine MS therapies were identified in the 24-month cohort (OCR, $n=524$ : oral, $n=701$; injectable, $n=365$; other IV, $n=120$ ), as shown in Fig. 2 and Supplementary Table 2 . The proportions of patients in each group, by index DMT, are shown in Supplementary Fig. 1. Baseline characteristics of the 24-month cohort are shown in Table 1. Notable differences in the baseline characteristics-consistent with the 12- and 18-month cohorts-included that patients initiating OCR were older (mean age [SD], 49 [10]), had higher CCI scores ( $17 \%$ categorized as $\geq 2$ ), were more likely to be male (33\%), and were more likely to have received a previous DMT (74\%) compared with other index DMT groups. 
Table 1 Baseline demographics and clinical characteristics for 24-month cohort $(N=1710)$

\begin{tabular}{|c|c|c|c|c|}
\hline & $\begin{array}{l}\text { OCR } \\
(n=524)\end{array}$ & $\begin{array}{l}\text { Oral } \\
(n=701)\end{array}$ & $\begin{array}{l}\text { Injectable } \\
(n=365)\end{array}$ & $\begin{array}{l}\text { Other IV } \\
(n=120)\end{array}$ \\
\hline Age at index, mean (SD), years & $49(10)$ & $46(11)$ & $45(12)$ & $43(11)$ \\
\hline \multicolumn{5}{|l|}{ Age category at index, $n$ (\%) } \\
\hline$<35$ years & $41(8)$ & $106(15)$ & $72(20)$ & $23(19)$ \\
\hline $35-44$ years & $115(22)$ & $195(28)$ & $98(27)$ & $44(37)$ \\
\hline $45-54$ years & $193(37)$ & $236(34)$ & $109(30)$ & $35(29)$ \\
\hline$\geq 55$ years & $175(33)$ & $164(23)$ & $86(24)$ & $18(15)$ \\
\hline \multicolumn{5}{|l|}{ Sex, $n(\%)$} \\
\hline Male & $171(33)$ & $160(23)$ & $76(21)$ & $25(21)$ \\
\hline Female & $353(67)$ & $541(77)$ & $289(79)$ & $95(79)$ \\
\hline \multicolumn{5}{|l|}{ Payer type, $n(\%)$} \\
\hline Commercial & $501(96)$ & $676(96)$ & $356(98)$ & $117(98)$ \\
\hline Medicare & $23(4)$ & $25(4)$ & $9(3)$ & $3(3)$ \\
\hline Pre-index relapse, $n(\%)$ & $201(38)$ & $200(29)$ & $115(32)$ & $53(44)$ \\
\hline Pre-index DMT use, $n$ (\%) & $386(74)$ & $359(51)$ & $84(23)$ & $56(47)$ \\
\hline \multicolumn{5}{|l|}{ CCI category, $n$ (\%) } \\
\hline 0 & $393(75)$ & $538(77)$ & $269(74)$ & $96(80)$ \\
\hline 1 & $44(8)$ & $85(12)$ & $54(15)$ & $8(7)$ \\
\hline$\geq 2$ & $87(17)$ & 78 (11) & $42(12)$ & $16(13)$ \\
\hline
\end{tabular}

$C C I$ Charlson Comorbidity Index, DMT disease-modifying therapy, $I V$ intravenous, $O C R$ ocrelizumab

A higher proportion of patients experienced $\geq 1$ pre-index relapse $(44 \%)$ in the other IV group.

Among patients with 24 months of post-index eligibility, the OCR group had a higher proportion of adherent and persistent patients compared with those initiating oral, injectable, and other IV DMTs. At 24 months, $80 \%$ of patients initiating OCR were adherent compared with those initiating oral (55\%), injectable (35\%), and other IV medications (54\%). In adjusted models, patients initiating oral, injectable, or other IV DMTs had higher relative risks (RRs) of non-adherence over 24 months (RR, 2.2 [95\% CI, 1.7-2.9]; RR, 3.0 [95\% CI, 2.2-4.0]; RR, 2.2 [95\% CI, 1.5-3.3], respectively; all $p<0.001$ ) (Fig. 3a). The mean PDC across the groups was $88 \%, 70 \%, 56 \%$, and $67 \%$ for OCR, oral, injectable, and other IV DMTs, respectively (Table 2). Similarly, $75 \%$ of patients receiving OCR were persistent on therapy compared with those initiating oral (54\%), injectable (33\%), and other IV DMTs (55\%). In adjusted models, patients initiating other groups of DMTs had greater adjusted RRs of discontinuation than those initiating OCR (oral: RR, 1.9 [95\% CI, 1.4-2.4]; injectable: RR, 2.5 [95\% CI, 1.9-3.4]; other IV: RR, 1.8 [95\% CI, 1.2-2.6]; all $p<0.001$ ) (Fig. 3b). Of the patients who discontinued in each group, $11 \%$ of patients switched from OCR to another DMT, while $24 \%, 30 \%$, and $48 \%$ switched from the index DMT to another DMT in the oral, injectable, and other IV groups, respectively (Table 3). 


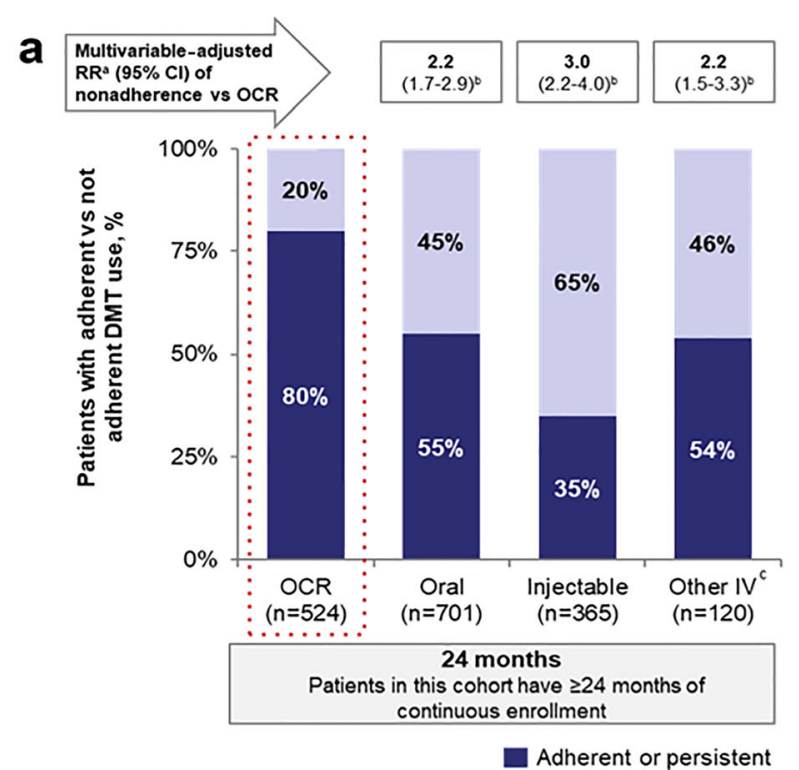

Fig. 3 Adherence to (a) and persistence with (b) OCR, injectable, oral, and other IV DMTs over 24 months. CCI Charlson Comorbidity Index, DMT disease-modifying therapy, $I V$ intravenous, $M S$ multiple sclerosis, $O C R$ ocrelizumab, $R R$ relative risk. ${ }^{a} \mathrm{RR}$ was estimated using a multivariable Poisson regression model adjusted for age,

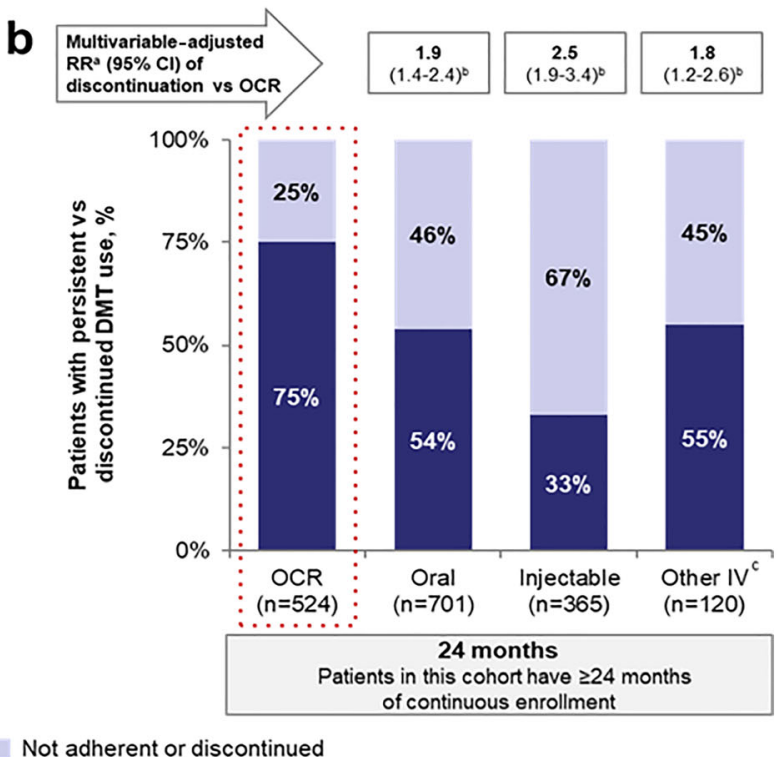

sex, index year, payer type (commercial vs. Medicare), insurance plan type, region, relapse in the prior year (yes/ no), CCI score, DMT use in the pre-index year (yes/no), and presence of MS symptoms (yes/no). ${ }^{\mathrm{b}} p<0.001$. ${ }^{c}$ Excludes alemtuzumab

Table 2 PDC measures at 24 months by route of administration

\begin{tabular}{lllll}
\hline \multicolumn{4}{l}{ Treatment group (24 months) } \\
\cline { 2 - 5 } & $\begin{array}{l}\text { OCR } \\
(\boldsymbol{n}=\mathbf{5 2 4})\end{array}$ & $\begin{array}{l}\text { Oral } \\
(\boldsymbol{n}=\mathbf{7 0 1})\end{array}$ & $\begin{array}{l}\text { Injectable } \\
(\boldsymbol{n}=\mathbf{3 6 5})\end{array}$ & $\begin{array}{l}\text { Other IV } \\
(\boldsymbol{n}=\mathbf{1 2 0})\end{array}$ \\
\hline Mean (SD) & $0.881(0.191)$ & $0.697(0.319)$ & $0.561(0.332)$ & $0.669(0.314)$ \\
Median & 0.967 & 0.858 & 0.537 & 0.843 \\
Range (min-max) & $0.029-1$ & $0.041-1$ & $0.036-1$ & $0.036-1$ \\
IQR (25th-75th percentile) & $0.896-0.986$ & $0.411-0.973$ & $0.23-0.923$ & $0.383-0.933$ \\
\hline
\end{tabular}

$I Q R$ interquartile range, $I V$ intravenous, $O C R$ ocrelizumab

At 12 and 18 months, those initiating other DMTs were between 3.2 and 4.6 times and 2.5 and 3.6 times more likely to be non-adherent compared with those initiating OCR, respectively (all $p<0.001$ ) (Fig. 4a). Likewise, those receiving other DMTs were 2.5 to 3.8 times and 2.0 to 3.0 times more likely to be non-persistent relative to OCR, respectively (all $p<0.001$ ) (Fig. 4b).

\section{Sensitivity Analysis}

When restricting the OCR patient cohort to those identified by permanent $\mathrm{J}$ or temporary codes, the 12- and 18-month results for adherence and persistence were similar to those of the full sample (Supplementary Table 3). Because the 24-month cohort had to have initiated the 
Table 3 Patients who discontinued at 24 months

\begin{tabular}{lllll}
\hline & \multicolumn{2}{l}{ Treatment group } & \\
\cline { 2 - 5 } & $\begin{array}{l}\text { OCR } \\
(\boldsymbol{n}=\mathbf{5 2 4})\end{array}$ & $\begin{array}{l}\text { Oral } \\
(\boldsymbol{n}=\mathbf{7 0 1})\end{array}$ & $\begin{array}{l}\text { Injectable } \\
(\boldsymbol{n}=\mathbf{3 6 5})\end{array}$ & $\begin{array}{l}\text { Other IV } \\
(\boldsymbol{n}=\mathbf{1 2 0})\end{array}$ \\
\hline No. of patients who discontinued & 129 & 324 & 244 & 54 \\
\% Discontinued (treatment gap) & 89.15 & 75.93 & 70.49 & 51.85 \\
\% Discontinued (switch) & 10.85 & 24.07 & 29.51 & 48.15 \\
\hline
\end{tabular}

$I V$ intravenous, $O C R$ ocrelizumab

DMTs in 2017, this sensitivity analysis was only performed for the patients receiving OCR in the 12- and 18-month cohorts.

RRs for likelihood of non-adherence and discontinuation at 12,18 , and 24 months relative to OCR were similar regardless of model choice when negative binomial models were performed in lieu of Poisson models to study adherence and persistence outcomes (Supplementary Table 4 ). Because of the binary nature of the outcome, all negative binomial models were under-dispersed, leading to estimates that were the same as those from the Poisson models.

\section{DISCUSSION}

The 24-month data highlight that OCR treatment was associated with better compliance compared with DMTs administered through other RoAs. Additionally, consistent with the previously published real-world analysis of OCR adherence and persistence [23], the results of this study confirmed that patients initiating OCR therapy are more likely to continue therapy after 12 and 18 months compared with those who initiate other MS DMTs. It was important to corroborate the results of the previous analysis through a separate large commercial data set, which included Medicare patients, to ensure reproducibility and generalizability of the first analysis. The OCR twiceyearly dosing schedule likely impacts a patient's ability to continue receiving therapy as prescribed compared with other more frequently administered therapies.
While these studies were largely similar in their designs, a few key differences should be highlighted for appropriate interpretation of the data. In the current analysis, excess days' supply was added to the coverage period in instances when patients refilled their prescription early for the oral and injectable groups. We adjusted this criterion, as early refills could increase the days covered and thereby potentially increase adherence rates for these RoAs. However, in instances when a patient received the infusion early (e.g., OCR or other IV DMT), the excess days in the dosing interval were not carried forward as there would not be additional days of coverage if an infusion was received earlier than due. This conservative approach would have benefited the oral and injectable groups by allowing overlap of prescription fills.

Furthermore, although the underlying cause is uncertain, the RRs of non-adherence and non-persistence were lower in the current assessment at both 12 and 18 months for all DMTs compared with the PharMetrics Plus analysis [23]. This may be a result of differences in samples within the databases but is likely the result of overlapping days of supply for oral and injectable DMTs being excluded in the PharMetrics analysis. As a result, the adherence and persistence rates would likely be higher for those RoAs in this analysis, thus dampening the RR.

Importantly, the adherence and persistence results for the other MS DMTs in this analysis were relatively similar to what has been reported in the published literature [12, 21, 32, 33]. A real-world analysis by Bergvall et al. [32] found 


\section{a}
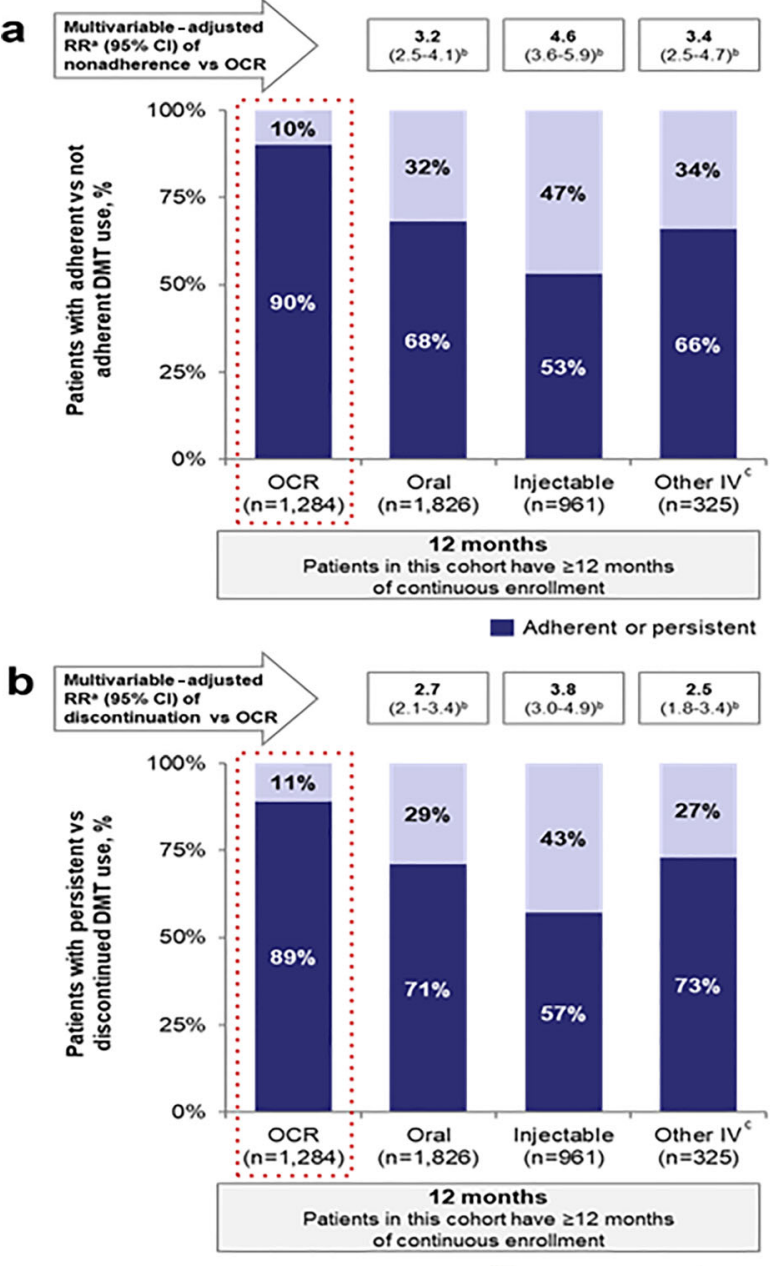

$\square$ Adherent or persistent

Fig. 4 Adherence to (a) and persistence with (b) OCR, injectable, oral, and other IV DMTs over 12 and 18 months. CCI Charlson Comorbidity Index, DMT disease-modifying therapy, $I V$ intravenous, $M S$ multiple sclerosis, $O C R$ ocrelizumab, $R R$ relative risk. ${ }^{a} \mathrm{RR}$ was estimated using a multivariable Poisson regression model

discontinuation rates of $27.9 \%$ for fingolimod (oral), 39.5\% for glatiramer acetate (injectable), $39.5 \%$ for natalizumab (other IV), and $43.7 \%$ for interferon (injectable) over 1 year. More recently, Duquette et al. [33] found the discontinuation rate of fingolimod to be $29 \%$ compared with $57 \%$ for injectable DMTs and $45 \%$ for natalizumab at 24 months $(p<0.001)$. The pooled reporting by RoA in our analysis does not allow direct comparison with individual DMTs, but the other IV (i.e., natalizumab)

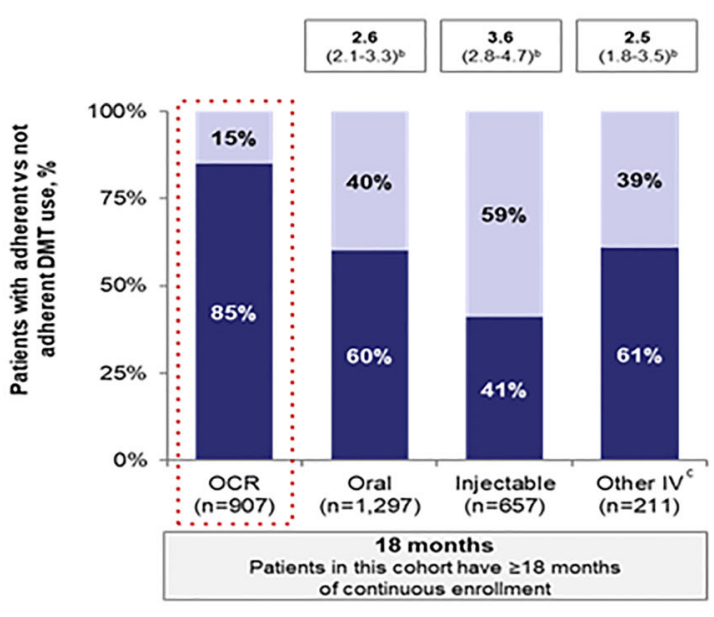

Not adherent or discontinued

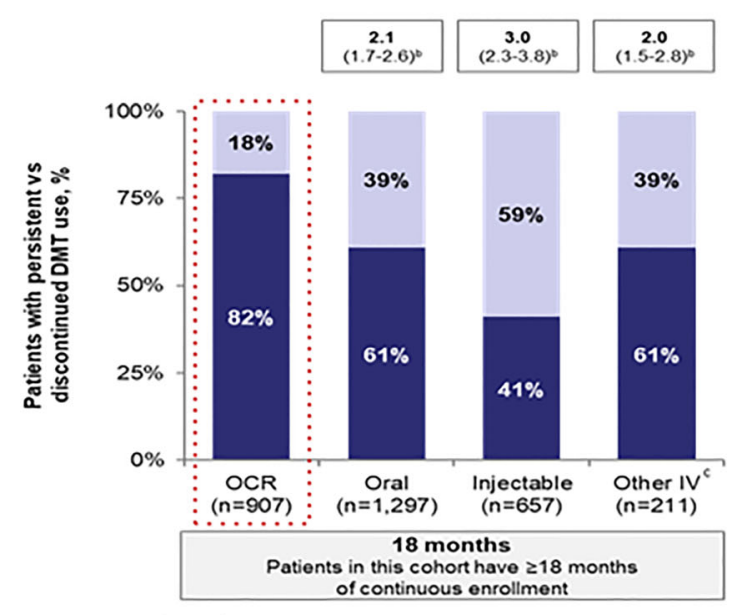

Not adherent or discontinued

adjusted for age, sex, index year, payer type (commercial vs. Medicare), insurance plan type, region, relapse in the prior year (yes/no), CCI, DMT use in the pre-index year (yes/ no), and presence of MS symptoms (yes/no). ${ }_{p}^{\mathrm{b}}<0.001$. ${ }^{c}$ Excludes alemtuzumab

discontinuation rate at 2 years is similar to that of Duquette et al. [33].

In this study we defined adherence using PDC to assess adherence and not the medication possession ratio. Both methods are credible, but PDC offers more reliable estimates of medication adherence, as the medication possession ratio can overestimate adherence, potentially resulting in a rate $>100 \%$. The PDC method is also now considered the adherence metric of choice for real-world studies and is 
endorsed by the Pharmacy Quality Alliance and the International Society for Pharmacoeconomics and Outcomes Research [28, 29].

Medication adherence continues to be a common issue for many chronic illnesses that require ongoing treatment [34-36]. Fortunately, the factors impacting adherence to and persistence of MS DMTs have been well established over the past two decades, with injection-related reasons (i.e., needle phobia, injection site reactions), forgetfulness, inconvenience, and perceived lack of efficacy being consistently cited [37-39]. Although the current study did not assess efficacy, good adherence has been correlated with higher efficacy treatment, such as reductions in relapse, resulting in lower healthcare resource utilization and cost [13]. In addition, the benefits observed with DMT adherence have been associated with improved work-related outcomes, such as reduced medical, sick leave, and short-term disability costs [40].

While optimizing medication adherence and persistence are vital to suppressing disease progression, a medication's attributes (e.g., safety, efficacy, RoA, convenience, etc.) have the potential to increase or decrease a patient's ability to comply with the therapy. OCR is a relatively new MS DMT; as clinicians gain realworld experience with it, sharing this real-world experience has provided the MS community with a more holistic view of the potential. Recently published real-world analyses highlight that OCR not only has a manageable safety profile as observed in randomized controlled trials but also is an effective treatment option $[41,42]$.

Maximizing compliance with DMTs is vital to ensuring that patients are benefiting from their prescribed treatment [39]. Clinicians should anticipate barriers and use any tools to mitigate non-compliance and reinforce the importance of taking the therapy as prescribed [43]. Given the common barriers to DMT compliance, OCR offers the advantage of being administered every 6 months after an initial loading dose, which may be a more suit- able choice for patients unable to receive more frequent DMTs.

To our knowledge, there is only one other published study [23] that compared the adherence and persistence of various DMTs with those of OCR. And while there is a growing body of literature assessing DMT compliance, there is a dearth of research looking into longerterm DMT compliance [33]. The studies conducted to date highlight that the RoA has an impact on adherence and persistence, yet the translation to clinical and humanistic outcomes requires further research.

\section{Limitations}

As with all claims analyses, there were limitations in our study. The adherence and persistence calculations were based on the dosing schedule per each product's prescribing information. As such, there may be instances in which the prescribing patterns differed from those of the FDA-approved labeling, leading to a misclassification of adherence and persistence. Another inherent limitation to claims data was that we were unable to ascertain whether patients prescribed injectable or oral DMTs actually consumed/administered the medication, thereby potentially overestimating adherence or persistence status.

Similarly, limiting the patient inclusion to those who had 12-, 18-, or 24-month follow-up data may have resulted in selection bias. However, sensitivity analyses (not shown) examining differences in adherence/persistence at 12 and 18 months between the 12- and 18-month cohorts and the 24-month cohort were small, suggesting that selection bias induced by these follow-up requirements was minimal. Moreover, claims data contain limited clinical information (e.g., MS subtype [relapsing-remitting vs. primary progressive] or line of therapy), and as OCR is the only therapy approved by the FDA for primary progressive MS, this may have resulted in confounding. Finally, we were unable to assess the rationale behind discontinuation and switches. 


\section{CONCLUSIONS}

This study confirms that patients initiating OCR in a real-world setting achieved superior adherence and persistence at 12,18 , and 24 months compared with those initiating other MS DMTs administered through other RoAs. As MS impacts patients in their peak productive years, slowing disease progression through improved medication adherence and persistence is a vital component of the holistic care patients should receive. Therefore, the challenges associated with medication compliance underscore the need for addressing the source of non-compliance and using appropriate mitigation strategies to reduce the negative consequences.

\section{ACKNOWLEDGEMENTS}

Funding. This study was funded by Genentech, Inc. (South San Francisco, CA, USA), a member of the Roche Group. Genentech is also funding the journal's Rapid Service Fee.

Editorial Assistance. Provided by Sarah Nordquist, PhD of Health Interactions (U.S) and funded by Genentech.

Authorship. All named authors meet the International Committee of Medical Journal Editors (ICMJE) criteria for authorship for this article, take responsibility for the integrity of the work as a whole, and have given their approval for this version to be published.

Author Contributions. GP made substantial contributions to the study conception; interpreted the data; and substantively revised the draft manuscript. EDP made substantial contributions to the conception; design of the work; the acquisition, analysis, and interpretation of data; and substantively revised the draft manuscript. CDN made substantial contributions to the conception; design of the work; the acquisition, analysis, and interpretation of data; and substantively revised the draft manuscript. KKB made substantial contributions to the study conception; analyzed and interpreted the data; and drafted the manuscript. DS made substantial contributions to the conception; design of the work; the acquisition, analysis, and interpretation of data; and substantively revised the draft manuscript. NGB made substantial contributions to the conception; design of the work; the acquisition, analysis, and interpretation of data; and substantively revised the draft manuscript.

Prior Presentation. At the American Academy of Neurology 2021 Virtual Annual Meeting; April 17-22, 2021. Presentation Number P15.228. Pineda E, et al. Adherence and Persistence to Disease-Modifying Therapies for Multiple Sclerosis and Their Impact on Clinical and Economic Outcomes in a US Claims Database.

Disclosures. Elmor D. Pineda, Nicole G. Bonnie, Daniel Sheinson, Komal K. Bawa, and Carmen D. Ng are employees of Genentech and shareholders of F. Hoffman-La Roche Ltd (Basel, Switzerland). Gabriel Pardo has served on advisory boards and/or speakers' bureau for Biogen Idec, Celgene/Bristol Myers Squibb, EMD Serono, Greenwich Biosciences, Janssen Pharmaceuticals, Novartis Pharmaceuticals, Roche/ Genentech, Sanofi-Genzyme, TG Therapeutics, VielaBio/Horizon Therapeutics.

Compliance with Ethics Guidelines. Data were de-identified and comply with the Health Insurance Portability and Accountability Act and the Helsinki Declaration and its later amendments or comparable ethical standards.

Data Availability. The IBM MarketScan Commercial and Medicare Supplemental claims data sets analyzed during this study are not publicly available as this is proprietary information. Qualified researchers may request access to individual patient-level data through the clinical study data request platform (https:// vivli.org/). Further details on Roche's criteria for eligible studies are available here (https://vivli. org/members/ourmembers/). For further details on Roche's Global Policy on the Sharing of Clinical Information and how to request access to related clinical study documents, see here (https://www.roche.com/research_and_develop 
ment/who_we_are_how_we_work/clinical_trials/ our_commitment_to_data_sharing.htm).

Open Access. This article is licensed under a Creative Commons Attribution-NonCommercial 4.0 International License, which permits any non-commercial use, sharing, adaptation, distribution and reproduction in any medium or format, as long as you give appropriate credit to the original author(s) and the source, provide a link to the Creative Commons licence, and indicate if changes were made. The images or other third party material in this article are included in the article's Creative Commons licence, unless indicated otherwise in a credit line to the material. If material is not included in the article's Creative Commons licence and your intended use is not permitted by statutory regulation or exceeds the permitted use, you will need to obtain permission directly from the copyright holder. To view a copy of this licence, visit http://creativecommons.org/licenses/by$\mathrm{nc} / 4.0 /$.

\section{REFERENCES}

1. Confavreux C, Vukusic S. The clinical course of multiple sclerosis. Handb Clin Neurol. 2014;122: 343-69.

2. Wallin MT, Culpepper WJ, Campbell JD, et al. The prevalence of MS in the United States. A population-based estimate using health claims data. Neurology. 2019;92:e1029-40.

3. National Multiple Sclerosis Society. Disease-modifying therapies for MS. http://www. nationalMSsociety.org/DMT. Accessed 7 Sep 2021.

4. Rogers JM, Panegyres PK. Cognitive impairment in multiple sclerosis: evidence-based analysis and recommendations. J Clin Neurosci. 2007;14:919-27.

5. Patten SB, Marrie RA, Carta MG. Depression in multiple sclerosis. Int Rev Psychiatry. 2017;29: 463-72.

6. Gil-González I, Martín-Rodríguez A, Conrad R, Pérez-San-Gregorio MÁ. Quality of life in adults with multiple sclerosis: a systematic review. BMJ Open. 2020;10: e041249.
7. Rae-Grant A, Day GS, Marrie RA, et al. Practice guideline recommendations summary: diseasemodifying therapies for adults with multiple sclerosis: report of the Guideline Development, Dissemination, and Implementation Subcommittee of the American Academy of Neurology. Neurology. 2018;90:777-88.

8. Katsarava Z, Ehlken B, Limmroth V, et al. Adherence and cost in multiple sclerosis patients treated with IM IFN beta-1a: impact of the CARE patient management program. BMC Neurol. 2015;15:170.

9. Irwin DE, Cappell KA, Davis BM, et al. Differences in multiple sclerosis relapse rates based on patient adherence, average daily dose, and persistence with disease-modifying therapy: observations based on real-world data. Value Health. 2015;18:A764.

10. Torkildsen O, Myhr KM, Bo L. Disease-modifying treatments for multiple sclerosis-a review of approved medications. Eur J Neurol. 2016;23(suppl 1):18-27.

11. Menzin J, Caon C, Nichols C, White LA, Friedman M, Pill MW. Narrative review of the literature on adherence to disease-modifying therapies among patients with multiple sclerosis. J Manage Care Pharm. 2013;19(1 suppl A):S24-40.

12. Johnson KM, Zhou H, Lin F, Ko JJ, Herrera V. Realworld adherence and persistence to oral diseasemodifying therapies in multiple sclerosis patients over 1 year. J Manage Care Spec Pharm. 2017;23: 844-52.

13. Burks J, Marshall TS, Ye X. Adherence to diseasemodifying therapies and its impact on relapse, health resource utilization, and costs among patients with multiple sclerosis. Clinicoecon Outcomes Res. 2017;9:251-60.

14. Yermakov S, Davis M, Calnan M, et al. Impact of increasing adherence to disease-modifying therapies on healthcare resource utilization and direct medical and indirect work loss costs for patients with multiple sclerosis. J Med Econ. 2015;18: 711-20.

15. Tan H, Cai Q, Agarwal S, Stephenson JJ, Kamat S. Impact of adherence to disease-modifying therapies on clinical and economic outcomes among patients with multiple sclerosis. Adv Ther. 2011;28:51-61.

16. Devonshire V, Lapierre $\mathrm{Y}$, Macdonell R, et al. The Global Adherence Project (GAP): a multicenter observational study on adherence to disease-modifying therapies in patients with relapsing-remitting multiple sclerosis. Eur J Neurol. 2011;18:69-77.

17. McQueen RB, Livingston $\mathrm{T}$, Vollmer $\mathrm{T}$, et al. Increased relapse activity for multiple sclerosis 
natalizumab users who become nonpersistent: a retrospective study. J Manage Care Spec Pharm. $2015 ; 21: 210-8$.

18. Thomas NP, Curkendall S, Farr AM, Yu E, Hurley D. The impact of persistence with therapy on inpatient admissions and emergency room visits in the US among patients with multiple sclerosis. J Med Econ. 2016;19:497-505.

19. Higuera L, Carlin CS, Anderson S. Adherence to disease-modifying therapies for multiple sclerosis. J Manage Care Spec Pharm. 2016;22:1394-401.

20. de Seze JBF, Brudon F. Patient perceptions of multiple sclerosis and its treatment. Patient Prefer Adher. 2012;6:263-73.

21. Nicholas JA, Edwards NC, Edwards RA, Dellarole A, Grosso M, Phillips AL. Real-world adherence to, and persistence with, once- and twice-daily oral diseasemodifying drugs in patients with multiple sclerosis: a systematic review and meta-analysis. BMC Neurol. 2020;20:281.

22. Tremlett H, Van der Mei I, Pittas F, et al. Adherence to the immunomodulatory drugs for multiple sclerosis: contrasting factors affect stopping drug and missing doses. Pharmacoepidemiol Drug Saf. 2008;17:565-76.

23. Engmann NJ, Sheinson D, Bawa K, Ng CD, Pardo G. Persistence and adherence to ocrelizumab compared with other disease-modifying therapies for multiple sclerosis in US commercial claims data. J Manage Care Spec Pharm. 2021;27:639-49.

24. Watson Health. IBM MarketScan Research Databases for Life Sciences Researchers - White Paper. https://www.ibm.com/downloads/cas/OWZWJOQO. Accessed 7 Sep 2021.

25. Engmann N, Yang E, Julian L, et al. Early experience with ocrelizumab: patients characteristics from a large insurance claims database. Presented at: 34th Congress of the European Committee for Treatment and Research in Multiple Sclerosis; 10-12 October 2018; Berlin, Germany. Abstract EP1706.

26. Boster A, Nicholas $\mathrm{J}, \mathrm{Wu} \mathrm{N}$, et al. Comparative effectiveness research of disease-modifying therapies for the management of multiple sclerosis: analysis of a large health insurance claims database. Neurol Ther. 2017;6:91-102.

27. Ollendorf DA, Jilinskaia E, Oleen-Burkey M. Clinical and economic impact of glatiramer acetate versus beta interferon therapy among patients with multiple sclerosis in a managed care population. J Manage Care Pharm. 2002;8:469-76.
28. Pharmacy Quality Alliance. PQA adherence measures. https://www.pqaalliance.org/adherencemeasures. Accessed 1 Jun 2021

29. Cramer JA, Roy A, Burrell A, et al. Medication compliance and persistence: terminology and definitions. Value Health. 2008;11:44-7.

30. Lenth RV, Buerkner P, Herve M, Love J, Riebl H, Singmann H. emmeans: estimated marginal means, aka least-squares means. https://cran.r-project.org/ package=emmeans. Accessed 28 May 2021.

31. HCPCS Codes. Temporary codes for use with outpatient prospective payment system. https://hcpcs. codes/c-codes/C9494/. Accessed 7 June 2021.

32. Bergvall N, Petrilla AA, Karkare SU, et al. Persistence with and adherence to fingolimod compared with other disease-modifying therapies for the treatment of multiple sclerosis: a retrospective US claims database analysis. J Med Econ. 2014;17:696-707.

33. Duquette P, Yeung M, Mouallif S, Nakhaipour HR, Haddad P, Schecter R. A retrospective claims analysis: compliance and discontinuation rates among Canadian patients with multiple sclerosis treated with disease-modifying therapies. PLoS One. 2019;14: e0210417.

34. Mody R, Huang Q, Yu M, et al. Adherence, persistence, glycaemic control and costs among patients with type 2 diabetes initiating dulaglutide compared with liraglutide or exenatide once weekly at 12-month follow-up in a real-world setting in the United States. Diabetes Obes Metab. 2019;21:920-9.

35. Mueller S, Wilke T, Bechtel B, Punekar YS, Mitzner $\mathrm{K}$, Virchow JC. Non-persistence and non-adherence to long-acting COPD medication therapy: a retrospective cohort study based on a large German claims dataset. Respir Med. 2017;122:1-11.

36. Clotet B, Carmena J, Pulido F, Luque I, RodriguezAlcántara F, COL30494 Cohort Study Members. Adherence, quality of life, and general satisfaction with co-formulated zidovudine, lamivudine, and abacavir on antiretroviral-experienced patients. HIV Clin Trials. 2004;5:33-9.

37. Treadaway K, Cutter G, Salter A, et al. Factors that influence adherence with disease-modifying therapy in MS. J Neurol. 2009;256:568-76.

38. Daugherty KK, Butler JS, Mattingly M, Ryan M. Factors leading patients to discontinue multiple sclerosis therapies. J Am Pharm Assoc. 2003;2005(45):371-5.

39. Patti F. Optimizing the benefit of multiple sclerosis therapy: the importance of treatment adherence. Patient Prefer Adher. 2010;4:1-9. 
40. Hersh CM, Brook RA, Beren IA, et al. The implications of suboptimal year-1 outcomes with diseasemodifying therapy in employees with multiple sclerosis. J Med Econ. 2021;24:479-86.

41. Cellerino M, Boffa G, Lapucci C et al. Predictors of ocrelizumab effectiveness in patients with multiple sclerosis. Neurotherapeutics (2021).
42. Fernandez-Diaz E, Perez-Vicente JA, VillaverdeGonzalez R, et al. Real-world experience of ocrelizumab in multiple sclerosis in a Spanish population. Ann Clin Transl Neurol. 2021;8(2):385-94.

43. Costello K, Kennedy P, Scanzillo J. Recognizing nonadherence in patients with multiple sclerosis and maintaining treatment adherence in the long term. Medscape J Med. 2008;10:225. 